\title{
Interaction and cystogenesis of Toxoplasma gondii within skeletal muscle cells in vitro
}

\author{
Erick Vaz Guimarães ${ }^{1}$, Laís de Carvalho² ${ }^{2}$, Helene Santos Barbosa ${ }^{1 /+}$ \\ 'Laboratório de Biologia Estrutural, Instituto Oswaldo Cruz-Fiocruz, Av. Brasil 4365, 21040-360 Rio de Janeiro, RJ, Brasil ²Laboratório de \\ Cultura de Células, Departamento de Histologia e Embriologia, Universidade do Estado do Rio de Janeiro, Rio de Janeiro, RJ, Brasil
}

\begin{abstract}
Infection by the protozoan parasite Toxoplasma gondii is widely prevalent in humans and animals. To prevent human infection, all meat should be well cooked before consumption, since the parasite is present in skeletal muscle. In this context, the use of skeletal muscle cells (SkMCs) as a cellular model opens up new approaches to investigate T. gondii-host cell interactions. Immunofluorescent detection of proteins that are stage-specific for bradyzoites indicated that complete cystogenesis of T. gondii in in vitro cultures of SkMCs occurs after $96 \mathrm{~h}$ of infection. Ultrastructural analysis showed that, after $48 \mathrm{~h}$ of interaction, there were alterations on the parasitophorous vacuole membrane, including greater thickness and increased electron density at the inner face of the membrane. The present study demonstrates the potential use of primary cultures of SkMCs to evaluate different molecular aspects of $\mathrm{T}$. gondii invasion and cystogenesis and presents a promising in vitro model for the screening of drug activities toward tissue cysts and bradyzoites.
\end{abstract}

Key words: Toxoplasma gondii - tissue cysts - bradyzoites - cystogenesis - skeletal muscle cells

The obligate intracellular pathogenic parasite Toxoplasma gondii, the causative agent of toxoplasmosis, has a life cycle that is comprised of three infective stages: tachyzoites, bradyzoites and sporozoites. These stages are found in feline infection (definitive hosts) and nonfeline infection (intermediate hosts). The two main transmission mechanisms of $T$. gondii infection are through ingestion of either: (i) oocysts shed into the environment from the faeces of cats or (ii) viable tissue cysts found in raw or undercooked meat of intermediate hosts (Dzierszinski et al. 2004, Ferguson 2004). In both intermediate and definitive hosts, the asexual cycle of development occurs within any nucleated cell of warm-blooded animals, such as mammals (including humans) and birds. This cycle consists of two infective stages: tachyzoites undergoing fast multiplication and bradyzoites, which are latent and undergo slow multiplication in tissue cysts during the chronic phase of toxoplasmosis. Tissue cysts are located mostly in the central nervous system, retina and in heart and skeletal muscles (Dubey 1993, 1998). In some species of intermediate hosts, the tissue cysts can persist for a lifetime, but this mechanism is still not fully understood (Tenter et al. 2000).

Normally, the immune response effectively prevents dissemination of this parasite, but sometimes a spontaneous reactivation of the latent infection occurs, char-

Financial support: CNPq, FAPERJ, FIOCRUZ (PAPES IV), IOC

+Corresponding author: helene@ioc.fiocruz.br

Received 10 October 2008

Accepted 2 February 2009 acterised by bradyzoite-tachyzoite differentiation. The interconversion process and subsequent parasite egress leads to infection of neighbouring cells and formation of new tissue cysts, thus maintaining the infection in the host (Montoya \& Liesenfeld 2004). Nevertheless, in immunocompromised hosts, such uncontrolled reactivation may be more frequent and result in a potentially fatal infection (Gazzinelli et al. 1993, Gross \& Pohl 1996). The disease has great importance in congenitally infected children that can present retinochoroiditis, hydrocephalus, convulsions and intracerebral calcification. The most common complication of congenital toxoplasmosis is ocular disease (Montoya \& Liesenfeld 2004). To prevent human infection, all meat should be well cooked before consumption (Hill \& Dubey 2002).

The $T$. gondii-host cell interaction involves an early stage of adhesion and posterior invasion of the host cell. These steps are both crucial for the establishment of parasite infection. The development and modification of the parasitophorous vacuole (PV), an interface between the parasite and host cell functions, facilitates replication and differentiation of the parasite, leading to its protection against free radicals, $\mathrm{pH}$ and osmolarity changes and the activation of the host immune system (Laliberté \& Carruthers 2008). Another advantage is that this vacuole does not fuse with the endocytic or exocytic membrane trafficking pathways of the host and fails to acidify (Joiner et al. 1990, Sinai 2008).

Limited attention has been given to the study of conversion and cystogenesis of $T$. gondii in skeletal muscle cells, although the parasite's preference for this cell type during chronic toxoplasmosis is well known (Andrade et al. 1999, 2001, Monteiro-Leal et al. 1998, Barbosa 1999, Barbosa et al. 1999, 2005, Ferreira-da-Silva et al. 2008, Guimarães et al. 2008). The implementation of skeletal muscle as a cellular model is justified because the ingestion of raw or undercooked meat containing tissue cysts 
is an important transmission route for toxoplasmosis. Furthermore, the process of cystogenesis is responsible for the maintenance of the infection.

\section{MATERIALS AND METHODS}

The procedures with animals were carried out in accordance with the guidelines established by the Fundação Oswaldo Cruz-Fiocruz Committee of Ethical for the Use of Animals by license CEUA 0229-04 and by the Guidelines on the Care and Use of Animals for Experimental Purposes (NACLAR).

Tissue cysts and bradyzoites isolation - T. gondii cysts from ME-49 strain (Type II) were maintained in C57BL/6 female mice (15-18 g) inoculated intraperitoneally with 50 cysts/animal. After four and 12 weeks postinfection, the mice were sacrificed and the brain cysts isolated, as described by Freyre (1995) and Popiel et al. (1996) and modified by Guimarães et al. (2007, 2008). To obtain intact bradyzoites from isolated tissue cysts, we employed the method developed by Popiel et al. (1996) and modified by Guimarães et al. (2008).

Primary culture of skeletal muscle cells (SkMCs) SkMC cultures were obtained from the thigh muscles of 18-day old mouse embryos. The cultures were maintained at $37^{\circ} \mathrm{C}$ for $4-5$ days to complete myogenesis and muscle fibre formation. During this period, the culture medium was renewed every two days according to Barbosa et al. (2000).

Fluorescence microscopy - Immunofluorescence assays were performed using specific monoclonal antibodies for bradyzoites (anti-BAG1-7E5). Briefly, bradyzoites were allowed to interact with SkMCs at a parasite-host cell ratio of 1:1. After 12-168 $\mathrm{h}$ of interaction, the cultures were fixed for $30 \mathrm{~min}$ at $\mathrm{rt}$ in $4 \%$ paraformaldehyde diluted in PBS, washed three times (10 min each) with PBS and then processed according to Guimarães et al. (2008). The samples were examined under a Nikon Eclipse E800 microscope. The images were obtained with a CoolSNAP-Pro colour camera and processed with the Image-Pro Plus 4.5.1.29 software in the Production and Image Management Sector/IOC-Fiocruz, RJ, Brazil. The monoclonal antibody anti-BAG1-7E5 was kindly presented by Dr. W. Bohne (Institut für Medizinische Mikrobiologie, Universität Göttingen, Germany).

Transmission electron microscopy - SkMCs were infected with $T$. gondii bradyzoites (1:1 parasite-host cell ratio) and, after variable periods of interaction (between $30 \mathrm{~min}$ at 31 days), the cultures were washed three times for $10 \mathrm{~min}$ with PBS and fixed for $1 \mathrm{~h}$ at $4^{\circ} \mathrm{C}$ in $2.5 \%$ glutaraldehyde diluted in $0.1 \mathrm{M}$ sodium cacodylate buffer containing $3.5 \%$ sucrose and $2.5 \mathrm{mM} \mathrm{CaCl}_{2}(\mathrm{pH} 7.2)$. After fixation, the cells were washed in the same buffer and then post-fixed for $30 \mathrm{~min}$ at $\mathrm{rt}$ in $1 \% \mathrm{OsO}_{4}$ diluted in $0.1 \mathrm{M}$ cacodylate buffer. The cells were washed in the same buffer, scraped from the plastic dish and centrifuged at $4^{\circ} \mathrm{C}$ for $5 \mathrm{~min}$ at $10,000 \mathrm{~g}$. Thereafter, the cells were dehydrated in a graded series of acetone and embedded in Epoxy resin. Thin sections were stained with uranyl acetate and lead citrate and then examined under a Zeiss EM10C transmission electron microscope.

\section{RESULTS}

The ultrastructural analysis of the skeletal muscle cell - T. gondii bradyzoite interaction showed parasites inside tight PVs after $30 \mathrm{~min}$ of infection (Fig. 1). After $48 \mathrm{~h}$ of infection, cells containing parasites in the endodyogeny process of cell division could be observed (Fig. 2). The first signs of changes in the structure of the PV membrane (PVM) were seen, including greater thickness and increased electron density at the inner face of the membrane, similar to the granular material found in the vacuole matrix. The presence of small membrane invaginations was also observed (Fig. 2). Finally, after 48 h, there were endoplasmic reticulum (ER) profiles of the host cell

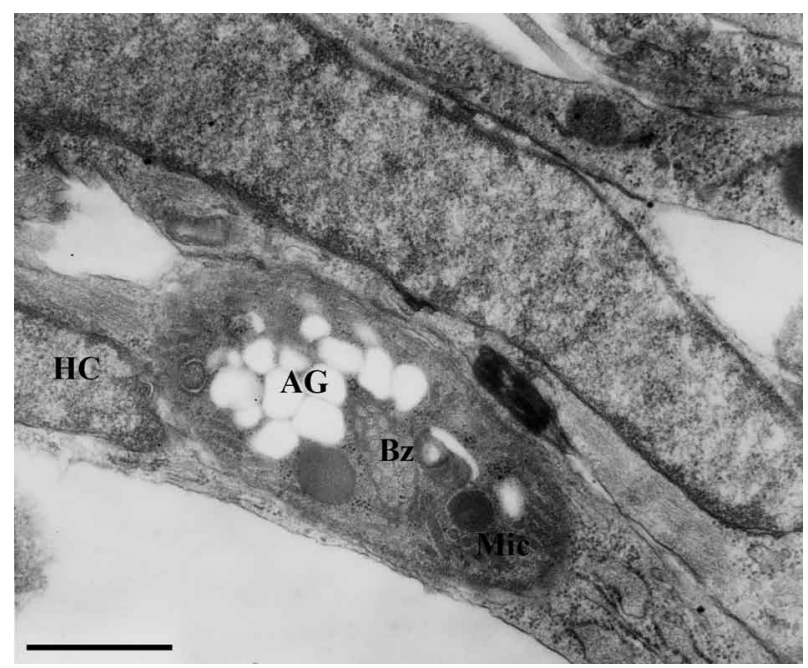

Fig. 1: ultrastructural analysis after $30 \mathrm{~min}$ of skeletal muscle cells-T. gondii bradyzoites interaction. Intracellular parasite were observed into parasitophorous vacuole. AG: amylopectin granules; Bz: bradyzoite; HC: host cell; Mic: micronemes. $\mathrm{Bar}=1 \mu \mathrm{m}$.

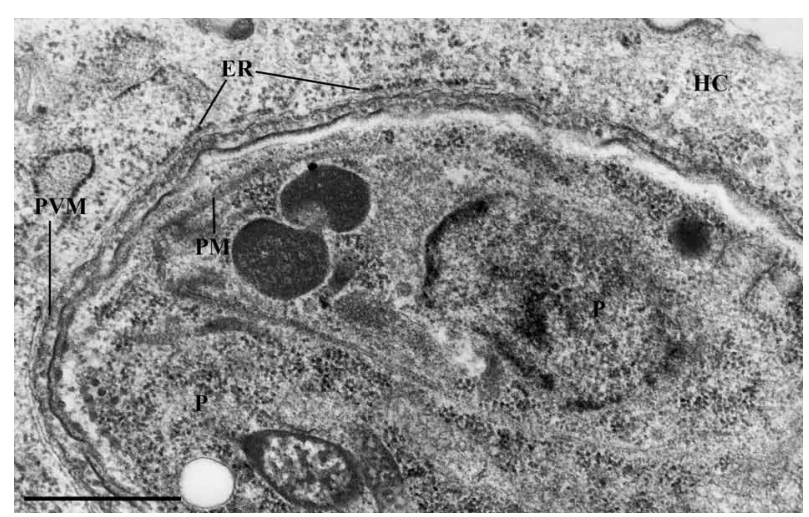

Fig. 2: transmission electron microscopy analysis of skeletal muscle cells after $48 \mathrm{~h}$ of Toxoplasma gondii bradyzoites infection. Parasitophorous vacuole membrane (PVM) presenting structural modifications to form the cyst wall, indicative that cystogenesis in vitro is occurring. Endoplasmic reticulum (ER) profiles were observed surrounding the parasitophorous vacuole that is under formation. HC: host cell; P: parasite; PM: plasma membrane. $\mathrm{Bar}=1 \mu \mathrm{m}$. 
around the PVM. The membrane face, which contained ribosomes, was turned toward the cytoplasm and the smooth face was in close contact with PVM (Fig. 2).

We monitored $T$. gondii interconversion within SkMCs by immunofluorescence, utilising an antibradyzoite (anti-BAG1) monoclonal antibody after 24 , 120 and $144 \mathrm{~h}$ of interaction. This analysis revealed few bradyzoites multiplying within mature myotubes (Figs 3-5). During the period of infection between 120-144 h, it was common to find both PVs that were anti-BAG1 positive and PVs that were anti-BAG1 negative for the same mature myotube. Although the infection has been exclusively with the bradyzoite form (Fig. 3), some parasites remained as bradyzoites, while others had become tachyzoites (Figs 4, 5).

After 31 days, the alterations observed in the PVM were consistent with wall cyst formation, including PVM thickening and the development of an electron dense granular region containing a large number of vesicles and tubules (Figs 6-8). These tissue cysts presented a dense matrix (Fig. 6) between the intracystic parasites. They showed a large amount of amylopectin granules, micronemes and electron-dense rhoptries with the nuclei localised in the posterior end, indicating that these parasites are typical bradyzoites (Figs 6, 7). Host cell organelles (ER profile and mitochondria) were rarely associated with the newly formed tissue cysts (Figs 6-8).

\section{DISCUSSION}

The majority of knowledge concerning the T. gondiihost cell interaction has been derived from in vitro assays with tachyzoites and there is little information in relation to the interaction with bradyzoite and sporozoite forms (Dubey et al. 1998, Fouts \& Boothroyd 2007).
Moreover, the majority of in vitro studies concerning the tachyzoite $\leftrightarrow$ bradyzoite conversion have been performed in cell lines for several reasons: (i) easy growth of these cells, (ii) long term survival as a monolayer and (iii) the type of cell line and/or the origin of the host cell are not thought to be decisive for tissue cyst formation in vitro (Lindsay et al. 1991, McHugh et al. 1993). This extrapolation has been based on results obtained with different cell lines and very rarely with primary cultures of the cells involved in the cystogenesis in vivo, for example, neural cells or muscle cells (Dubey et al. 1998). Consequently, experimental assays using skeletal muscle cells (a target cell where the parasite is resident during the chronic phase of the disease) to investigate cystogenesis in vitro, or even the parasite biology or other aspects of the parasite-host cell interaction, are limited to studies carried out by our group (Andrade et al. 1999, 2001, Barbosa et al. 2005, Ferreira-da-Silva et al. 2008, Guimarães et al. 2008).

For many protozoan parasites, differentiation (interconversion) is an obligate process, with different evolutionary stages within its life cycle that is necessary for the success of parasite transmission (Dzierszinski et al. 2004). The maintenance of $T$. gondii in tissues is crucial to establish the chronic infection and also is probably responsible for toxoplasmosis reactivation. This is determined by the host immunosuppression, which leads to disruption of tissue cysts and subsequent bradyzoitetachyzoite conversion (Lyons et al. 2002). A hypothesis was postulated linking the protective role of neuroglobin and myoglobin to the cytotoxic effect of nitric oxide, which is also observed during infection with Plasmodium and Trypanosoma cruzi in red blood cells and cardiomyocytes, respectively (Taylor-Robinson, 1998, Ascenzi et al. 2001, 2005). In this context, these results
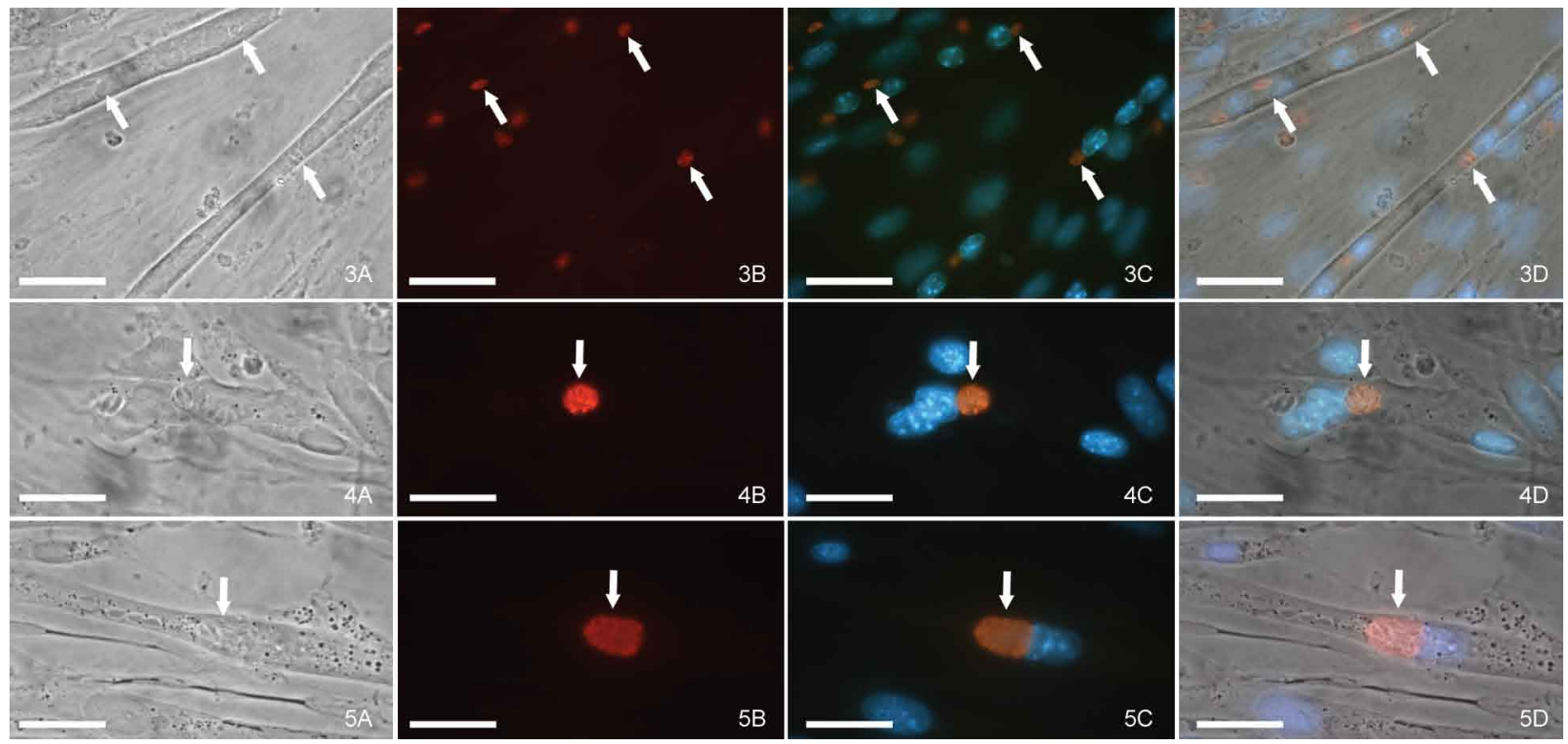

Figs 3-5: immunofluorescence assays showing skeletal muscle cells after 24, 120 and $144 \mathrm{~h}$ of infection with bradyzoite forms, respectively. A: phase contrast microscopy of infected myotube; B: bradyzoites (arrow) inside parasitophorous vacuole were labeled with anti-BAG1; C: double labeling of the infected culture with DAPI plus anti-BAG1; D: merge showing phase contrast microscopy, nuclei (DAPI - blue) and anti-BAG1 (red). Bar $=20 \mu \mathrm{m}$. 
could justify the persistence of $T$. gondii cysts in retina, in the nervous system and heart and skeletal muscles for a long time. The bradyzoite-tachyzoite interconversion in SkMCs, as shown by immunofluorescence assays, was expected since immune system components that are
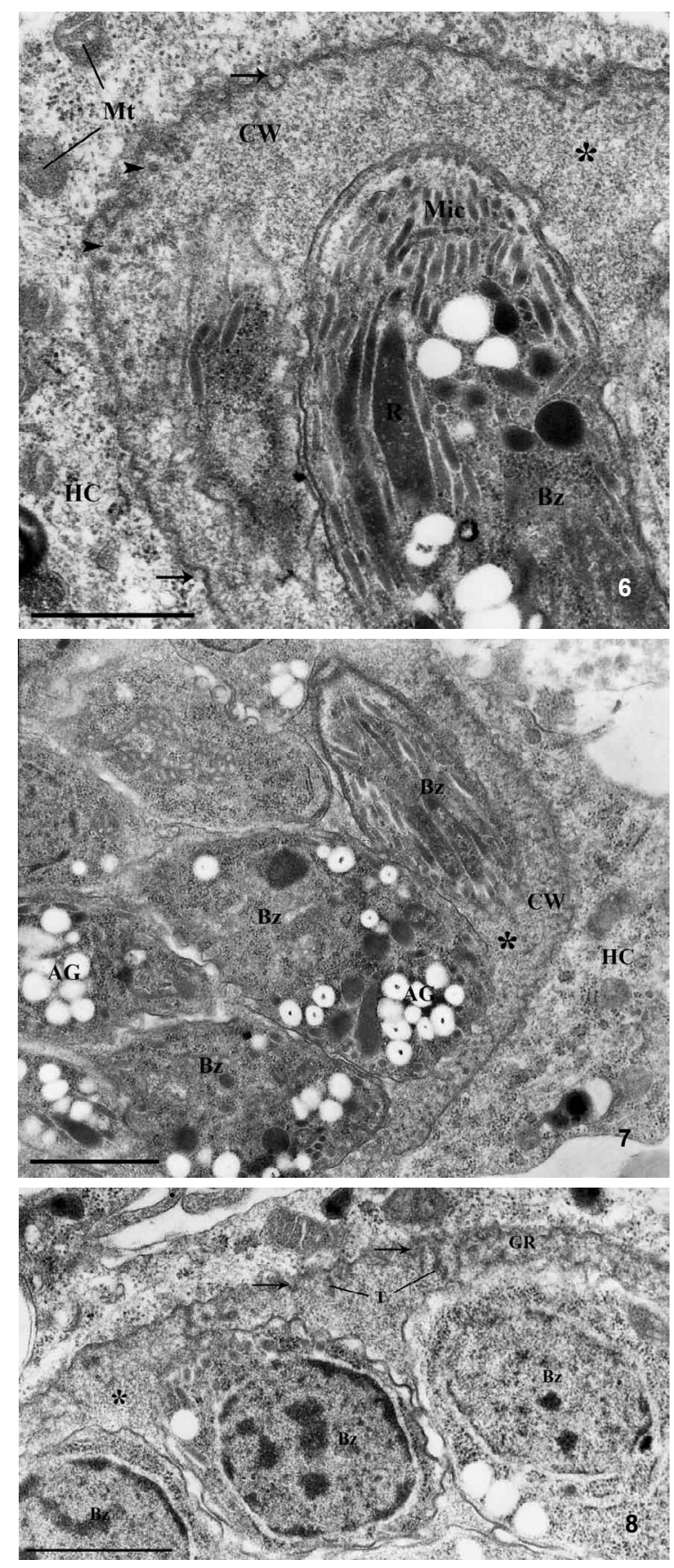

Figs 6-8: transmission electron microscopy analysis of skeletal muscle cells after 31 days of infection with Toxoplasma gondii bradyzoites. Besides having many typical bradyzoites (Bz), the cyst wall (CW) can be characterized by membrane invaginations (arrow) and tubules $(\mathrm{T})$ in the granular region (GR). Rare profiles of endoplasmic reticulum or mitochondria (Mt) associated with the cyst wall were observed. AG: amylopectin granules; HC: host cell; Mic: micronemes; $\mathrm{R}$ : rhoptries; asterisk: cyst matrix. $\mathrm{Bar}=1 \mu \mathrm{m}$. responsible for the control of tachyzoite proliferation in the in vivo system were not present in the in vitro system. The strategies that we employed to overcome this variable were to keep a low parasite-host cell ratio, to monitor the intracellular development for up to 31 days and to observe complete cystogenesis, in vitro.

Unlike the large amount of data on tachyzoite infection during the parasite-host cell interaction, little is known concerning the changes caused by bradyzoite infection. To understand these differences it is necessary to analyse bradyzoite infection at the ultrastructural and molecular level (Sasono \& Smith 1998, Fouts \& Boothroyd 2007). Thus, the use of primary culture of SkMCs as host cells, and of the bradyzoite form of the parasite, allowed us to partially mimic the in vivo infection system, since ingestion of raw or undercooked meat is one of the main routes of infection by $T$. gondii (Weiss \& Kim 2000).

The formation of typical tissue cysts in our in vitro culture system clearly shows the potential of this system in the study of $T$. gondii cystogenesis. Thus, this cellular model may be useful for several approaches, including: (i) monitoring and evaluating intracellular $T$. gondii development, (ii) characterising the molecular mechanisms involved in cyst formation within the host cells and (iii) analysing the action of anti-parasitic drugs against tissue cysts and bradyzoites of $T$. gondii, considering that the current therapies act on tachyzoites and that such treatments have little or no effect on the wall tissue cyst and bradyzoites. Current therapies act on tachyzoites and such treatments have little or no effect on the wall tissue cyst or bradyzoites.

\section{ACKNOWLEDGEMENTS}

To Sandra Maria de Oliveira Souza, Marielle Moreira and Genesio Lopes, for their technical assistance, to David Straker, for the English revision, and to Dr. Wolfgang Bohne (Institut für Medizinische Mikrobiologie, Universität Göttingen, Germany), who supplied the monoclonal antibodie anti-BAG1.

\section{REFERENCES}

Andrade EF, Carvalho L, Barbosa HS 1999. Association of lipids during Toxoplasma gondii-skeletal muscle cells interaction. Acta Microscopy 8: 423-424.

Andrade EF, Stumbo AC, Monteiro-Leal LH, Carvalho L, Barbosa HS 2001. Do microtubules around the Toxoplasma gondii-containing parasitophorous vacuole in skeletal muscle cells form a barrier for the phagolysosomal fusion? J Submicrosc Cytol Pathol 33: 337-341.

Ascenzi P, Bocedi A, Gradoni L 2005. Do neuroglobin and myoglobin protect Toxoplasma gondii from nitrosative stress? IUBMB Life 57: 689-691.

Ascenzi P, Salvati L, Brunori M 2001. Does myoglobin protect Trypanosoma cruzi from the antiparasitic effects of nitric oxide? FEBS Lett 501: 103-105.

Barbosa HS 1999. Why studies on invasion of host cell by Trypanosoma cruzi using established cell lines or primary cell cultures give conflicting results? Mem Inst Oswaldo Cruz 94 (Suppl I): 153-154.

Barbosa HS, Pereira MCS, Meirelles MNL 2000. Protocolos de culturas primárias. In TC Araújo-Jorge, SL de Castro, Doença de Chagas: Manual para experimentação animal, Fundação Oswaldo Cruz, Rio de Janeiro, p. 297-313. 
Barbosa HS, Silva MFF, Guimaraes EV, Carvalho L, Rodrigues RM 2005. Absence of vacuolar membrane involving Toxoplasma gondii during its intranuclear localization. J Parasitol 91: 182-184.

Dubey JP 1993. Toxoplasma, Neospora, Sarcocystis and other tissue cyst-forming coccidian of humans and animals. In JP Kreier, Parasitic protozoa, Vol. VI, Academic Press Inc, New York, p. 1-158.

Dubey JP 1998. Advances in the life cycle of Toxoplasma gondii. Int J Parasitol 28: 1019-1024.

Dubey JP, Lindsay DS, Speer CA 1998. Structures of Toxoplasma gondii tachyzoites, bradyzoites and sporozoites and biology and development of tissue cysts. Clin Microbiol Rev 11: 267-299.

Dzierszinski F, Nishi M, Ouko L, Roos DS 2004. Dynamics of Toxoplasma gondii differentiation. Eukaryot Cell 3: 992-1003.

Ferguson DJ 2004. Use of molecular and ultrastructural markers to evaluate stage conversion of Toxoplasma gondii in both the intermediate and definitive host. Int J Parasitol 34: 347-360.

Ferreira-da-Silva MF, Barbosa HS, Gross U, Lüder CGK 2008. Stressrelated and spontaneous stage differentiation of Toxoplasma gondii. Molecular Biosystems 4: 1-11

Fouts AE, Boothroyd JC 2007. Infection with Toxoplasma gondii bradyzoites has a diminished impact on host transcript levels relative to tachyzoite infection. Infect Immun 75: 634-642.

Freyre A 1995. Separation of Toxoplasma cysts from brain tissue and liberation of viable bradyzoites. J Parasitol 81: 1008-1010.

Gazzinelli RT, Denkers EY, Sher A 1993. Host resistance to Toxoplasma gondii: model for studying the selective induction of cellmediated immunity by intracellular parasites. Infect Agents Dis 2: $139-149$.

Gross U, Pohl F 1996. Influence of antimicrobial agents on replication and stage conversion of Toxoplasma gondii. Curr Top Microbiol Immunol 219: 235-245.

Guimaraes EV, Acquarone M, De Carvalho L, Barbosa HS 2007. Anionic sites on Toxoplasma gondii tissue cyst wall: expression, uptake and characterization. Micron 38: 651-658.

Guimaraes EV, De Carvalho L, Barbosa HS 2008. Primary culture of skeletal muscle cells as a model for studies of Toxoplasma gondii cystogenesis. J Parasitology 94: 72-83.
Hill D, Dubey JP 2002. Toxoplasma gondii: transmission, diagnosis and prevention. Clin Microbiol Infect 8: 634-640.

Joiner KA, Fuhrman SA, Miettinen HM, Kasper LH, Mellman I 1990. Toxoplasma gondii: fusion competence of parasitophorous vacuoles in Fc receptor-transfected fibroblasts. Science 249: 641-646.

Laliberté J, Carruthers VB 2008. Host cell manipulation by the human pathogen Toxoplasma gondii. Cell Mol Life Sci 65: 1900-1915.

Lindsay DS, Dubey JP, Blagburn BL, Toivio-Kinnucan M 1991. Examination of tissue cyst formation by Toxoplasma gondii in cell cultures using bradyzoites, tachyzoites and sporozoites. $J$ Parasitol 77: 126-132.

Lyons RE, McLeod R, Roberts CW 2002. Toxoplasma gondii tachyzoite-bradyzoite interconversion. Trends Parasitol 18: 198-201.

McHugh TD, Gbewonyo A, Johnson JD, Holliman RE, Butcher PD 1993. Development of an in vitro model of Toxoplasma gondii cyst formation. FEMS Microbiol Lett 114: 325-332.

Monteiro-Leal, LH, Stumbo AC, Campanati L, Barbosa HS, Carvalho L 1998. Video-microscopy analysis of the interaction of Toxoplasma gondii with host cells. Mem Inst Oswaldo Cruz 93 (Suppl. II): 110.

Montoya JG, Liesenfeld O 2004. Toxoplasmosis. Lancet 363: 19651976.

Popiel I, Gold MC, Booth KS 1996. Quantification of Toxoplasma gondii bradyzoites. J Parasitol 82: 330-332.

Sasono PM, Smith JE 1998. Toxoplasma gondii: an ultrastructural study of host-cell invasion by the bradyzoite stage. Parasitol Res 84: 640-645.

Sinai AP 2008. Biogenesis of and activities at the Toxoplasma gondii parasitophorous vacuole membrane. Subcell Biochem 47: 155-164.

Taylor-Robinson AW 1998. Nitric oxide can be released as well as scavenged by hemoglobin: relevance to its antimalarial activity. Parasite Immunol 20: 49-50.

Tenter AM, Heckeroth AR, Weiss LM 2000. Toxoplasma gondii: from animals to humans. Int J Parasitol 30: 1217-1258.

Weiss LM, Kim K 2000. The development and biology of bradyzoites of Toxoplasma gondii. Front Biosci 5: D391-405. 